\title{
Examining the central and peripheral processes of written word production through meta-analysis
}

\author{
Jeremy J. Purcell ${ }^{1}$, Peter E. Turkeltaub ${ }^{2}$, Guinevere F. Eden ${ }^{1}$ and Brenda Rapp ${ }^{3}$ * \\ ${ }^{1}$ Department of Pediatrics, Center for the Study of Learning, Georgetown University, Washington, DC, USA \\ 2 Department of Neurology, Georgetown University, Washington, DC, USA \\ ${ }^{3}$ Department of Cognitive Science, Johns Hopkins University, Baltimore, MD, USA
}

\section{Edited by:}

Albert Costa, University Pompeu

Fabra, Spain

Reviewed by:

Pelagie M. Beeson, University of

Arizona, USA

Steven Z. Rapcsak, University of

Arizona, USA

${ }^{*}$ Correspondence:

Brenda Rapp, Department of Cognitive Science, Johns Hopkins

University, 135 Krieger Hall, 3400

North Charles Street, Baltimore, MD

21218-2685, USA.

e-mail: rapp@cogsci.jhu.edu
Producing written words requires "central" cognitive processes (such as orthographic longterm and working memory) as well as more peripheral processes responsible for generating the motor actions needed for producing written words in a variety of formats (handwriting, typing, etc.). In recent years, various functional neuroimaging studies have examined the neural substrates underlying the central and peripheral processes of written word production. This study provides the first quantitative meta-analysis of these studies by applying activation likelihood estimation (ALE) methods (Turkeltaub et al., 2002). For alphabet languages, we identified 11 studies (with a total of 17 experimental contrasts) that had been designed to isolate central and/or peripheral processes of word spelling (total number of participants $=146$ ). Three ALE meta-analyses were carried out. One involved the complete set of 17 contrasts; two others were applied to subsets of contrasts to distinguish the neural substrates of central from peripheral processes. These analyses identified a network of brain regions reliably associated with the central and peripheral processes of word spelling. Among the many significant results, is the finding that the regions with the greatest correspondence across studies were in the left inferior temporal/fusiform gyri and left inferior frontal gyrus. Furthermore, although the angular gyrus (AG) has traditionally been identified as a key site within the written word production network, none of the metaanalyses found it to be a consistent site of activation, identifying instead a region just superior/medial to the left AG in the left posterior intraparietal sulcus. These meta-analyses and the discussion of results provide a valuable foundation upon which future studies that examine the neural basis of written word production can build.

Keywords: spelling, writing, meta-analysis, fMRI, dysgraphia, fusiform gyrus, intraparietal sulcus, angular

\section{INTRODUCTION}

There are a number of reasons why it is important to understand the neural substrates of written language production. Communicating through written language is critically important to professional success and for effective functioning in everyday life (e.g., writing checks, keeping a calendar, taking messages, etc.). In this regard, its importance has only increased with the rise of electronic communication (e-mail, internet, texting, etc.). As a result, deficits of written communication have a very significant impact on the well-being of individuals who suffer from acquired and developmental dysgraphia. Understanding the neural substrates of written language production is important for developing accurate prognoses and effective remediation of these written language impairments. Furthermore, written language processing is an interesting domain from a basic neurobiological perspective. Written language is a relatively recent human invention, appearing approximately 5000 years ago and used by only a limited portion of the human population until very recently. As a result, it is unlikely to have had an impact on the human genome and, accordingly, there is unlikely to be a genetic blueprint for the specific neural circuitry of written language processing. Nonetheless, with instruction, most people learn to comprehend and produce written language with remarkable ease. Thus, written language offers an opportunity to investigate the brain's capacity to develop expertise in skills that are not specifically biologically predetermined.

While in the past two decades there has been a great deal of functional neuroimaging research directed at understanding the brain-basis of written language comprehension (reading), relatively little attention has been directed at investigating written language production (spelling and writing). Recently, however there has been an upswing in the number of functional neuroimaging investigations in this domain. The findings from these studies, along with those from the more traditional clinical literature examining correlations between lesions and deficits, have provided important insights into the neurobiology of written language production. The neuroimaging studies, quite naturally, differ with regard to a number of variables such as experimental and control tasks, neuroimaging modalities, etc. This heterogeneity, as well as the current critical mass of functional neuroimaging studies of spelling, makes this an appropriate moment to attempt to integrate findings across studies. In this paper, we report on our efforts to do so by carrying out a meta-analysis of existing positron 
emission tomography (PET) and functional magnetic resonance imaging (fMRI) studies of word spelling in alphabetic language involving adult participants.

Producing written words involves a number of interacting cognitive processes that have been described in various models of written language production (Roeltgen and Heilman, 1985; Rapp and Caramazza, 1997; Rapcsak and Beeson, 2002; Hillis and Rapp, 2004). Although these cognitive processes are highly integrated, an important distinction is often made between central and peripheral components (see Figure 1). The different patterns of impairment that have been observed in cases of acquired dysgraphia subsequent to brain lesions have constituted the major source of empirical support for the distinctions between central and peripheral processing components as well as for the more finegrained distinctions described below and depicted in Figure 1. In addition, convergent evidence for many of these distinctions has been confirmed by behavioral studies of spelling and writing in neurologically healthy participants. While it is outside the scope of this paper to review these literatures, we refer the interested reader to various reviews (Ellis, 1979; Burt and Fury, 2000; Burt and Tate, 2002; Weingarten, 2005).

Spelling typically begins by hearing words (e.g., taking notes in a lecture, a message over the phone, etc.) or with internally generated word meanings (e.g., writing a letter, a grocery list, etc.). These auditory comprehension and semantic processes and mechanisms are not specific to spelling, yet serve as the basis for the subsequent retrieval or assembly of spellings. Spelling-specific, central processes are usually identified as: orthographic long-term memory (O-LTM; the orthographic lexicon), phoneme-grapheme (PG) conversion, and orthographic working memory (the graphemic buffer). O-LTM is the store of the word spellings that an individual is familiar with. As indicated in Figure 1, information in O-LTM may be retrieved on the basis of a word's meaning or, according to some researchers, directly from a representation of the word's sound (Patterson, 1986). In addition to retrieval from O-LTM, word spellings may be assembled from a phonological stimulus via the $\mathrm{PG}$ conversion processes that apply learned information regarding the relationships between sounds and letters (or other sub-lexical units) to generate plausible spellings for sound strings. For example, the sound stimulus "wuns" could result in the retrieval of the information O-N-C-E from O-LTM and/or in the assembly of a plausible spelling such as $\mathrm{W}-\mathrm{U}-\mathrm{N}-\mathrm{S}-\mathrm{E}$ from the PG conversion system. The letter representations assembled or retrieved are assumed to be abstract, lacking format-specific information (such as shape, size, motor plan, etc.). The abstract letter strings are then processed by $\mathrm{O}$ WM, a limited capacity system responsible for maintaining letter identity and order information active so that they can be selected for further processing by peripheral components (Rapp and Kong, 2002; Kan et al., 2006). These central processes interact with one another, with evidence specifically supporting bi-directional interactions between O-WM and O-LTM (McCloskey et al., 2006) and between O-LTM and PG conversion processes (Rapp et al., 2002).

In terms of peripheral processes, it is generally assumed that there are multiple stages involved in going from the abstract letters representations in $\mathrm{O}-\mathrm{WM}$ to the correct ordering and execution of the effector-specific muscle movements required for expressing

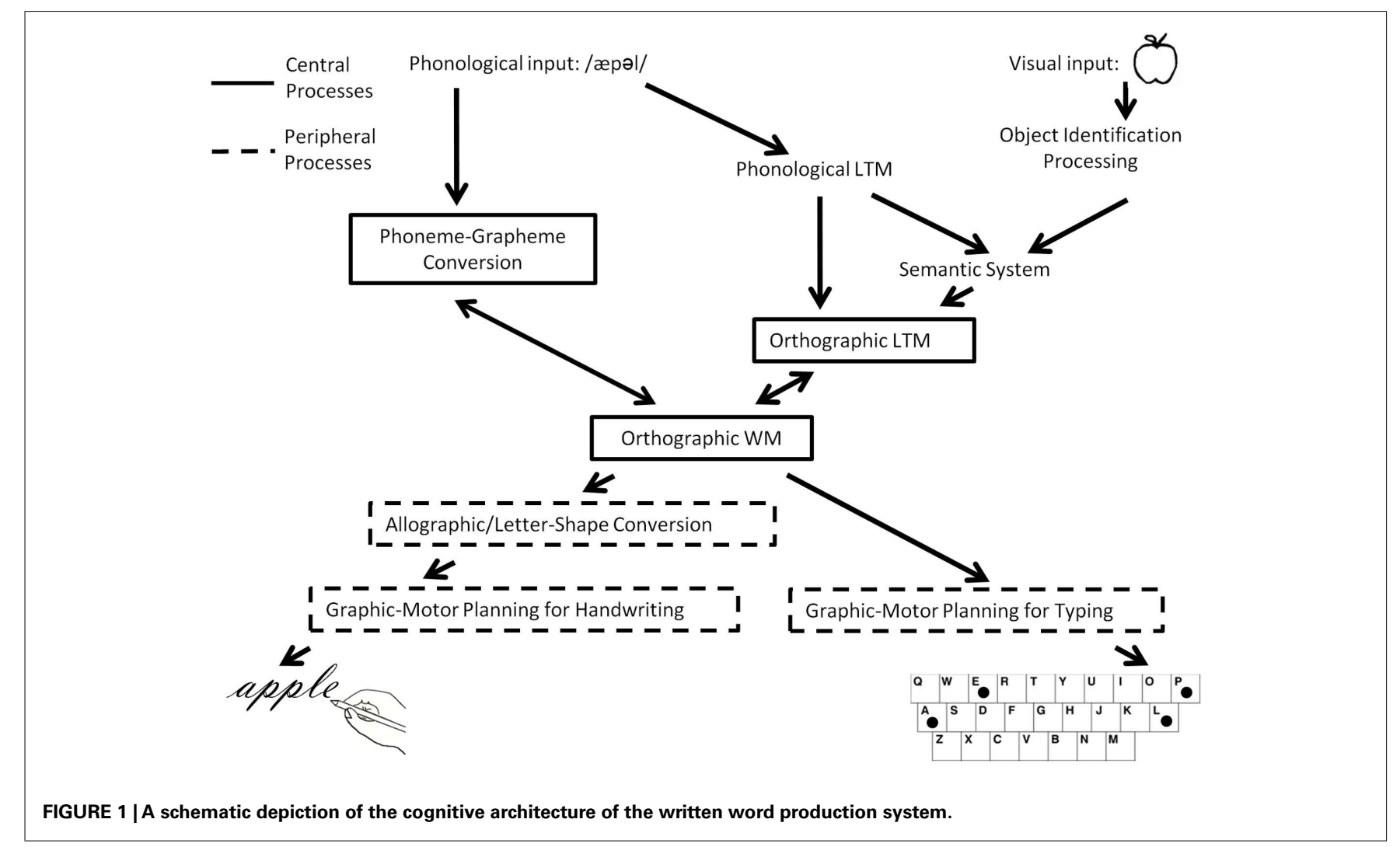


these letters. These peripheral processes generate written language in the major modalities of oral spelling, written spelling, or typing. As of yet there is no strong consensus on the characterization of the multiple peripheral processes, and so we present a general overview of some of the basic components (Ellis, 1979, 1982; Margolin, 1984; Ellis and Young, 1988; Rapp and Caramazza, 1997).

While we will not be concerned with oral spelling in this paper (as there have been no functional neuroimaging studies of oral spelling), it is worth mentioning the peripheral processes that are involved in this spelling "format." On the basis of the information held in O-WM, the names of letters (e.g., /si//ei//ti/) are retrieved in their correct order from the phonological LTM store of words (the phonological lexicon). Subsequently, the phonetic and articulatory planning processes of speech are recruited for the spoken production of the letter names.

In the case of writing, the specific letter forms or allographs must first be selected (upper-case, lower case, print, cursive, etc.). The mechanism responsible for converting the abstract letters held in O-WM to specific letter shapes is often referred to as Allographic or Letter-Shape Conversion. Following Allographic Conversion, motor plans for producing the letter forms are then assembled by Graphic Motor Planning processes, specifying such things as the size and ordering of the strokes. Subsequently, Effector-Specific Motor Programming compiles instructions for the specific limb/s (e.g., right or left hand, foot, etc.) to be used in carrying out the motor actions. The programming and execution of effectorspecific actions is subject to multi-modal feedback during execution (haptic, tactile, visual, etc.). In addition, some researchers have posited buffers/WM components that are associated with motor planning and programming components (Ellis and Young, 1988). Furthermore, the various motor planning processes necessarily interact with others responsible for certain spatial aspects of the writing process such as the coordination of the ego-centric and allo-centric reference frames required for producing the specific motor acts in the correct spatial locations.

In contrast to writing, typing requires the generation of a series of hand and finger motor commands in standard keyboard space in order to generate the letters held in O-WM. If we assume an experienced typist, motor plans may be stored and/or pre-compiled not only for individual letters but also for larger graphemic chunks, allowing for rapid production of letter sequences. Motor planning in typing is sensitive to the particular sequencing of letters, with the planning of the timing of hand movements for multiple letters typically occurring in parallel. As a result, the movements required to produce each letter are modulated by the immediately preceding and following context; this is sometimes referred to as co-articulation (West and Sabban, 1982; Salthouse, 1986; Salthouse and Saults, 1987). The motor programs used in typing also require parameterization for such things as speed of typing, size of keyboard, etc. As is the case for writing, the motor planning and execution processes required for typing also interact with processes that ensure the coordination of the spatial frames of reference required to move the hands and fingers in external keyboard space.

Handwriting and typing face a number of similar computational demands and, as a result, possibly share processing components. For example, motor sequences for both typing and handwriting have been shown to be similarly influenced (in terms of timing of strokes and pauses) by the types of grapheme units being produced (digraphs, double letters, syllable, and morpheme boundaries; Weingarten et al., 2004). Some investigators have even posited that handwriting and typing may share buffering or working memory components (Magrassi et al., 2010). For example, recent work has reported similar impairments to handwritten and typed production, but not oral spelling, subsequent to electrical stimulation to a restricted area of the superior parietal lobe (SPL; Magrassi et al., 2010).

Turning to the neural correlates of these central and peripheral components, findings from numerous studies of acquired dysgraphia subsequent to neural injury (typically stroke, but also surgical resection and progressive neurological disease) consistently indicate that the central processes of spelling are subserved by a network of regions including the left fusiform/inferior temporal, middle temporal, superior temporal, inferior frontal, angular, and supramarginal gyri (Beauvois and Derouesne, 1981; Shallice, 1981; Roeltgen and Heilman, 1984; Kawahata et al., 1988; Rapcsak and Beeson, 2004; Philipose et al., 2007; Rapcsak et al., 2009; Tsapkini and Rapp, 2010). In the lesion-based literature, peripheral processes have been most consistently associated with a left frontal-parietal network including, but not limited to, the dorsal premotor cortex and SPL (Exner, 1881; Ritaccio et al., 1992; Tohgi et al., 1995; Lubrano et al., 2004; Roux et al., 2009). In addition, peripheral dysgraphias have been described that are associated with damage to neural regions involved in general motor processing (not specifically in written language production) such as motor cortex, cerebellum, and various sub-cortical structures, e.g., caudate, putamen, and thalamus (Tanridag and Kirshner, 1985; Pramstaller and Marsden, 1996; Denes et al., 2005; Fournier Del Castillo et al., 2011). Finally, it is worth mentioning that spatial dysgraphias have been associated with some forms of spatial neglect (e.g., Caramazza and Hillis, 1990).

Although the lesion literature has provided critical information regarding the necessary substrates for written language production, it is subject to the well-known challenges of large and uncontrolled lesions and concerns regarding functional reorganization. In particular, precise localizations are typically quite difficult. Thus, lesion studies are well-complemented by functional neuroimaging techniques in non-impaired, healthy individuals. This effort has been strengthened in recent years by numerous fMRI or PET studies that have examined all or some component processes of written language production.

In general, functional neuroimaging studies of written production can be grouped into two types; those that make use of tasks that involve only central processing and those that make use of tasks that combine both central and peripheral processing. For instance the "central-only" tasks are those that require subjects to access the spelling of a word, but which do not involve writing (or typing). The "central + peripheral" tasks on the other hand, are those that require subjects to write or type words. In studies using central + peripheral tasks, central and peripheral processing components can sometimes be distinguished from one another depending on the additional experimental conditions employed and the specific contrasts they afford. We discuss these issues is more detail in the Section "Methods." 
With regard to central processes, the neuroimaging literature has generally supported the findings of the lesion literature that the left fusiform/inferior temporal gyrus (ITG), inferior frontal gyrus (IFG), and supramarginal gyrus (SMG) are associated with the central processes of spelling. As would be expected, activations in these regions have been reported for tasks involving either only central processing (Booth et al., 2002, 2004; Rapp and Lipka, 2011), or both central and peripheral processing (Beeson et al., 2003; Purcell et al., 2011). Interestingly, the few neuroimaging studies that have examined both reading and spelling within the same study have identified overlapping activations in both the left fusiform/ITG and IFG, suggesting that the these substrates in particular are important for central processes shared by both spelling and reading (Purcell et al., 2011; Rapp and Lipka, 2011). In addition the left SMG has also been associated with central processes (Petrides et al., 1995; Booth et al., 2002; Beeson et al., 2003; Purcell et al., 2011). Specifically, it has primarily been associated with sub-lexical (PG conversion) processing. This association is supported by the observation of SMG activation in studies of Japanese Kana writing, which is thought to rely heavily on PG conversion processing (Katanoda et al., 2001; Sugihara et al., 2006).

One apparent contradiction between lesion and functional neuroimaging studies concerns the left angular gyrus (AG). Although the lesion literature has long suggested it plays a role in central processes of written production, this region has not been consistently identified in the neuroimaging literature. While some studies have reported activation in this region (Booth et al., 2002, 2003a), a number of others have not (Beeson et al., 2003; Purcell et al., 2011). Furthermore, some of the latter studies have reported clusters in the intraparietal sulcus (IPS) region superior and medial to the left AG (Beeson et al., 2003; Purcell et al., 2011). Given the heterogeneity of these findings, the role of the posterior parietal cortex in written language production, and in particular with regard to the central processes, merits more careful scrutiny.

With regard to the peripheral components of written language production, in the neuroimaging literature these have primarily been associated with a left dorsal premotor/superior parietal network, as well as with activity in the cerebellum and sub-cortical structures such as the basal ganglia and thalamus (Menon and Desmond, 2001; Beeson et al., 2003; Sugihara et al., 2006; Roux et al., 2009; Purcell et al., 2011). The left dorsal premotor cortex in particular has been considered relevant to writing-specific processes involving the generation of graphemic motor commands (Menon and Desmond, 2001; Beeson et al., 2003). Although some of the earlier neuroimaging work in this field suggested that the left dorsal premotor region was associated with the generation of handwriting-specific graphic motor commands (Beeson et al., 2003), recent findings indicate that this general region also plays a role in the generation of graphic motor commands of typed production (Purcell et al., 2011).

Although a qualitative narrative summary such as the one presented above is valuable, it does not allow for a precise localization of the shared activations reported across studies. Metaanalytic methods allow us to address these challenges by quantitatively identifying brain locations that are consistently associated with tasks or cognitive functions of interest. Therefore, we applied the activation likelihood estimation (ALE) technique (GingerALE 2.1a3, BrainMap.org) to the study of written word production. The ALE technique is a widely used, validated, automated, quantitative method for a voxel-wise meta-analysis of neuroimaging foci which has been used in a range of cognitive domains such as reading (Turkeltaub et al., 2002), speech perception (Turkeltaub and Coslett, 2011), and object naming (Price et al., 2005).

Briefly, the goal of the ALE approach is to estimate, for each voxel in a normalized brain, the likelihood that it corresponds to the peak of a significant cluster in a task/contrast of interest. The logic underlying the approach is that, although significant activations are reported as discrete $X, Y, Z$ locations, there is uncertainty regarding their precise location. This uncertainty can be modeled as a three-dimensional Gaussian probability density distribution around the activation peaks that have been reported for a study. By combining the probability distributions corresponding to all of the significant activation peaks from all of the contributing studies, and then applying appropriate statistical corrections and thresholds, the ALE algorithm estimates the likelihood that a voxel corresponds to a location of peak activity in the literature. This analysis yields "clusters" of significant activation likelihood estimates that represent the spatial overlap of peak activity among the contributing studies.

In this paper, we report on the results of a series of metaanalyses. In the first, we applied the ALE algorithm to the findings of 11 written language production neuroimaging studies with a combined total of 17 separate contrasts. We then analyzed two subsets of the contrasts separately to identify central-only components of the spelling process and central + peripheral components. Finally, we compared the results of central + peripheral to centralonly ALE analyses in order to identify neural substrates that are reliably associated with the peripheral processes of written production. In combination, this set of analyses allowed us to identify the brain regions that are most reliably associated with central and peripheral written language production processes in alphabetic writing.

\section{METHODS}

\section{SELECTION OF STUDIES}

We searched Pubmed and Googlescholar online databases for studies associated with written language production using keywords "writing," "handwriting," "spelling," "orthographic," "fMRI," "PET," and "neuroimaging" in relevant combinations. Reference lists for appropriate publications were also searched for additional studies that could be included. Direct e-mail communication with some researchers also provided additional data sets for analysis.

We included studies based on the following inclusion criteria: (1) the neuroimaging technique used was fMRI or PET; (2) subjects were neurologically healthy, right-handed adults; (3) experiments required participants to generate orthographic lexical and/or sub-lexical representations; (4) studies involved an alphabetic written language; (5) no visual word or non-word stimuli were presented during the task of interest (in order to allow us to more clearly distinguish spelling from reading processes); (6) results were reported in a stereotactic three-dimensional 
coordinate system; (7) the field of view covered the whole brain; (8) analyses were not restricted to regions of interest; (9) a random-effects analysis was performed.

\section{SELECTION AND CATEGORIZATION OF CONTRASTS}

We assigned the selected studies to either central-only $(C)$ or central and peripheral $(\mathrm{C}+\mathrm{P})$ categories. More precisely, we placed the selected contrasts into one of these two categories. This ensured that the meta-analyses would be based not simply on the characteristics of the experimental tasks, but rather on the cognitive processes that were "isolated" on the basis of the contrast performed. The C-contrasts were those that predominantly isolated central processing components, whereas the $\mathrm{C}+\mathrm{P}$ contrasts were those that included both central and substantive peripheral processes.

An example of a C contrast is the one reported in Rapp and Lipka (2011). In this study, the experimental task required subjects to view a letter, listen to an auditorily presented word and then respond with a button press if the visually presented letter was in the spelling of the auditorily presented word. This task requires recruitment of O-LTM, and possibly PG conversion, as well as $\mathrm{O}-\mathrm{WM}$, but does not engage peripheral spelling processes. The contrasting (baseline) task was a perceptual-motor control task that allowed for the visual, auditory, motor, and decision components of the experimental task to be "subtracted out" in the contrast. An example of a $\mathrm{C}+\mathrm{P}$ contrast comes from Beeson et al. (2003). In this study, the experimental task required subjects to think of and handwrite words from a specific category (e.g., fruits) during a fixed time period. The baseline task was a circle-drawing task that served to exclude the most general peripheral motor aspects of handwriting, while retaining not only the central processes but also many of the writing-specific peripheral components.

Some of the studies that met the inclusion criteria for the metaanalysis included multiple contrasts. For example, the Beeson et al. (2003) study, in addition to a word writing task, included a circledrawing task as well as an alphabet writing task. For the latter task, subjects had to write as much of the alphabet as possible during a limited time period (comparable to the time period for writing words). While the word writing vs. circle-drawing contrast provided information regarding both central + peripheral processes, the word writing vs. alphabet writing contrast served to more specifically isolate central processes. In this way, some studies contributed multiple sets of contrasts and their corresponding results to the meta-analysis.

\section{ALE METHODS}

The $X, Y, Z$ coordinates of every significant peak (or subpeak) for all eligible contrasts constituted the input to the meta-analysis. Coordinates that were reported in Talairach space (Talairach and Tournoux, 1988) were converted to Montreal Neurological Institute (MNI) coordinates (Lancaster et al., 2007). The ALE meta-analysis was implemented using GingerALE 2.1a3 (www.brainmap.org). A new ALE algorithm was employed which consists of three steps resulting in an ALE map that is unbiased by the number of foci or the number of contrasts included from each study (Turkeltaub et al., 2011).
The three steps of the ALE analyses are as follows: (1) localization uncertainty is modeled for each focus of activity as a Gaussian, the width of which is determined from the number of subjects of the study (Eickhoff et al., 2005); (2) taking the union of the study-specific localization probabilities identified for each voxel yields the voxel-wise ALE value; (3) significance is tested using a random-effects method with a null hypothesis that the location of activation in each study is independent of the others (Eickhoff et al., 2009). The ALE maps generated according to steps 1-3 were thresholded with a False Discovery Rate of $q>0.05$ and clusters size $>100 \mathrm{~mm}^{3}$. For each of the resulting significant clusters, we additionally considered the number of studies that contributed to each. We did so by tallying the studies with foci within $2 \mathrm{SD}$ of localization uncertainty (see Eickhoff et al., 2009). In order to ensure that the reported results represented coherence across multiple experiments, we eliminated ALE clusters and peaks that were based on fewer than three different contrasts.

Three ALE analyses were carried out: the "All-Contrasts" analysis included all of the eligible contrasts, the "C-only" analysis included all eligible $\mathrm{C}$-contrasts and the " $\mathrm{C}+\mathrm{P}$ " analysis included all of the eligible $\mathrm{C}+\mathrm{P}$ contrasts. Additionally, we performed a subtraction analysis to identify locations where the $\mathrm{C}+\mathrm{P}$ ALE values were significantly greater than C-only ALE values, thus providing a means for identifying regions associated with peripheral processing. This subtraction analysis simply subtracts two ALE maps generated from two different groups of studies. Significance is tested via a permutation method which reassigns the studies randomly into two groups of the same size as the original ones over 5000 iterations. The distribution of ALE values in these random ALE subtraction maps provides a null hypothesis for the significance test.

Visualizations of the results were implemented with MRIcron, using the Colin brain template in MNI space (Holmes et al., 1998). Surface renderings are maximum intensity projections with a search depth of $16 \mathrm{~mm}$. Gyral anatomical labels were assigned based on the automated anatomical labeling (AAL) atlas developed for SPM (Tzourio-Mazoyer et al., 2002). Brodmann areas (BAs) were identified from the template developed for MRIcron. Activation likelihood peaks which were found deep in the gray matter at the sulcus boundaries between two gyri were assigned to the appropriate sulcus name. For instance, if the maximum probability label at a given peak was the SPL and the corresponding cluster was located deep in the gray matter of the IPS (which defines the boundary of the AG and the SPL), then a label of superior parietal lobule/intraparietal sulcus (SPL/IPS) was used in the tables and in the text.

\section{RESULTS}

\section{IDENTIFICATION OF ELIGIBLE STUDIES/CONTRASTS}

A total of 17 experimental contrasts were identified in 11 different publications which met our inclusion and exclusion criteria for the All-Contrasts meta-analysis and together represented a total of 146 subjects. All but one study was conducted using fMRI and all employed block designs except for one which employed an eventrelated design (Cohen et al., 2004). Most studies were conducted with English speaking participants, with two of the 11 studies performed with French native speakers. The eligible contrasts with a 
brief description of the tasks, the number of subjects, the language and imaging modality are listed in Table $\mathbf{1 .}$

\section{ANALYSIS 1: ALL-CONTRASTS}

As reported in Table 2 and Figure 2A, the ALE analysis identified 16 significant clusters with their corresponding peaks, as well as six additional significant sub-peaks. The peaks correspond to the voxel with the highest ALE value within a cluster of significant ALE values. Sub-peaks are the non-maximal peaks within clusters that have higher ALE values than their surrounding voxels. It is important to emphasize that the results reported in the table and figure do not correspond to the activation maps that are typically reported in functional neuroimaging. Instead, all significant voxels identified by the ALE analysis correspond to locations where, considering the peak coordinates identified by the set of eligible studies, an activation peak is likely to occur.

The ALE locations identified in the first analysis were primarily in the left hemisphere. In the left frontal lobe, the analysis identified four ALE clusters with the peaks located in the left posterior IFG, the superior frontal gyrus/sulcus, the supplementary

Table 1 | Meta-analysis dataset.

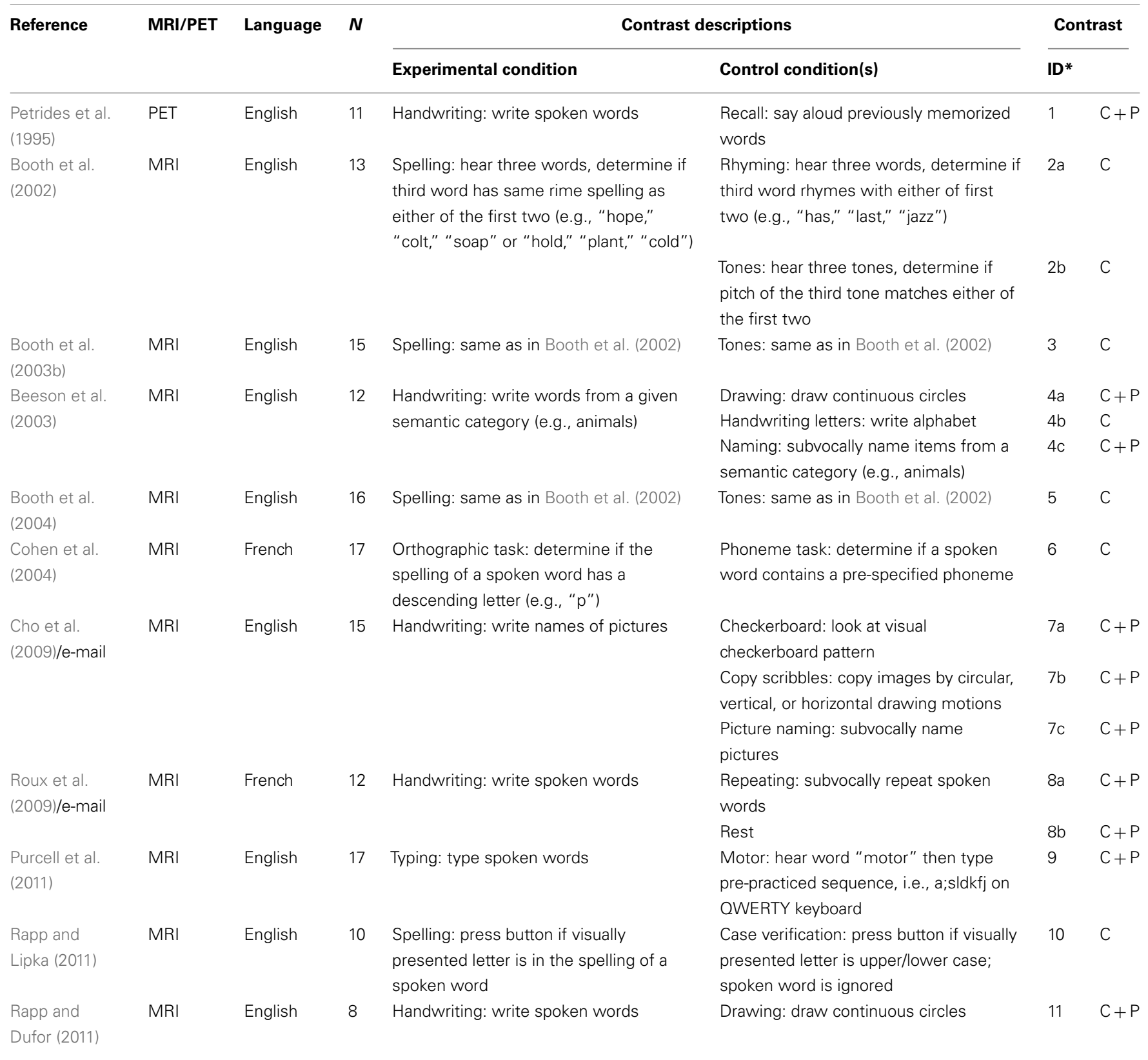

*The contrast ID letters designate tasks associated with each study. The $C+P$ and $C$ label denotes whether the task involved was either a central + peripheral (C $+P$ ) or central-only (C) task. 
Table 2 | Results for the all-contrasts written production meta-analysis.

\begin{tabular}{|c|c|c|c|c|c|c|c|c|c|c|}
\hline \multicolumn{2}{|c|}{ Location (Brodmann area) } & \multirow[t]{2}{*}{ Extent $\left(\mathrm{mm}^{3}\right)$} & \multicolumn{3}{|c|}{ Peak MNI } & \multicolumn{3}{|c|}{ Peak TAL } & \multirow{2}{*}{$\begin{array}{l}\text { ALE value } \\
\left(\times 10^{-3}\right)\end{array}$} & \multirow{2}{*}{$\begin{array}{l}\text { Contrasts contributing } \\
\text { to peaks }\end{array}$} \\
\hline & & & $x$ & $\boldsymbol{Y}$ & $Z$ & $x$ & $Y$ & $z$ & & \\
\hline \multicolumn{11}{|c|}{ FRONTAL } \\
\hline \multirow[t]{2}{*}{ Left } & IFG (9) & 3456 & -46 & 16 & 18 & -44 & 12 & 21 & 19.3 & $2 a, 3,4 b, 5$ \\
\hline & IFG (9) & - & -44 & 6 & 28 & -42 & 2 & 29 & 15.0 & $2 b, 9,10,11$ \\
\hline Left & SMA (6) & - & -4 & -10 & 50 & -5 & -15 & 48 & 15.7 & $1,4 c, 7 a, 11$ \\
\hline Left & SMA (6) & 1544 & -4 & 22 & 46 & -5 & 15 & 47 & 20.3 & $2 a, 4 a, 4 b, 5,9$ \\
\hline \multirow[t]{2}{*}{ Left } & Precentral gyrus (4) & 1120 & -36 & -24 & 60 & -35 & -29 & 55 & 15.2 & $1,7 a, 7 c, 8 a, 8 b$ \\
\hline & Postcentral gyrus (3) & - & -30 & -24 & 48 & -29 & -28 & 44 & 11.3 & $4 b, 7 a, 7 c$ \\
\hline \multirow[t]{2}{*}{ Right } & Insula (13) & 1144 & 42 & 24 & -4 & 38 & 21 & 3 & 15.2 & $4 a, 4 b, 7 b, 9$ \\
\hline & Insula (13) & - & 36 & 26 & -8 & 32 & 23 & 0 & 13.6 & $4 a, 4 b, 5,7 b$ \\
\hline Left & SMG (40) & 704 & -52 & -32 & 34 & -50 & -34 & 31 & 14.4 & $1,2 b, 7 c$ \\
\hline \multicolumn{11}{|c|}{ TEMPORAL } \\
\hline Left & STG/STS (21) & 1512 & -60 & -12 & -2 & -57 & -12 & 0 & 29.5 & $2 a, 3,5,9,11$ \\
\hline Right & STG/STS (21) & 728 & 52 & -12 & -6 & 47 & -13 & -2 & 17.3 & $2 a, 3,5,11$ \\
\hline \multicolumn{11}{|c|}{ OCCIPITOTEMPORAL } \\
\hline \multirow[t]{3}{*}{ Left } & ITG (37) & 4024 & -50 & -60 & -16 & -47 & -56 & -16 & 26.5 & $2 a, 2 b, 3,4 a, 4 b, 7 a, 9,10$ \\
\hline & FG (37) & - & -44 & -56 & -12 & -42 & -53 & -12 & 22.9 & $2 b, 4 a, 4 b, 6,7 a, 7 b, 9,10$ \\
\hline & ITG (37) & - & -42 & -58 & -6 & -40 & -55 & -7 & 20.3 & $2 b, 4 a, 6,7 a, 7 b, 11$ \\
\hline \multicolumn{11}{|c|}{ SUB-CORTICAL } \\
\hline Left & Thalamus & 744 & -14 & -10 & 10 & -14 & -12 & 12 & 13.0 & $7 a, 7 c, 11$ \\
\hline Left & Putamen & 576 & -24 & -4 & -2 & -23 & -5 & 2 & 15.6 & $4 a, 7 a, 7 c$ \\
\hline
\end{tabular}

motor area (SMA), and the fourth straddling the pre and postcentral gyri. In the parietal lobe there were three clusters: two were located in the superior parietal lobule and one in the SMG. In the left temporal lobe there was one cluster in the middle portion of the superior temporal gyrus/sulcus. In the occipitotemporal region there was one large cluster that included peaks in the ITG as well as one in the mid-fusiform gyrus (FG). In addition to these cortical locations in the left hemisphere, sub-cortical ALE clusters were identified in the thalamus and putamen. In the right hemisphere, there was a significant cluster in the insula, the posterior superior temporal gyrus/sulcus as well as three clusters in the right cerebellum.

\section{ANALYSIS 2: CENTRAL (C)-CONTRASTS}

For the C-contrasts analysis, a total of seven contrasts were identified which met our inclusion and exclusion criteria (Table 1). These contrasts were derived from six different publications and represent a total of 83 subjects.

As reported in Table 3 and Figure 2B, the C-contrasts ALE analysis identified six significant clusters with their corresponding peaks, and one additional sub-peak. All but one of the clusters were in the left hemisphere, and all were cortical. In the left frontal lobe there were two clusters: one large cluster in the posterior IFG and another in the anterior cingulate cortex. In the parietal lobe there was a single cluster in the superior parietal lobule/intraparietal sulcus. In the temporal lobe there was one cluster in the left superior temporal gyrus/superior temporal sulcus (STG/STS). Finally, in the occipitotemporal area there was a large cluster in ITG that extended into the FG. In the right hemisphere, only one ALE cluster was identified in the superior temporal gyrus/sulcus.

\section{ANALYSIS 3: CENTRAL + PERIPHERAL $(C+P)$ CONTRASTS}

For the $\mathrm{C}+\mathrm{P}$ Contrasts analysis, a total of 10 experimental contrasts were identified which met our inclusion and exclusion criteria (Table 1). These contrasts were derived from 6 different papers and included a total of 75 subjects.

As reported in Table 3 and Figure 2C, the ALE analysis identified eight significant clusters with their corresponding peaks, and five additional sub-peaks. Clusters were primarily in the left hemisphere, with only three right hemisphere clusters. In the left frontal lobe there was one cluster in the superior frontal gyrus/sulcus 


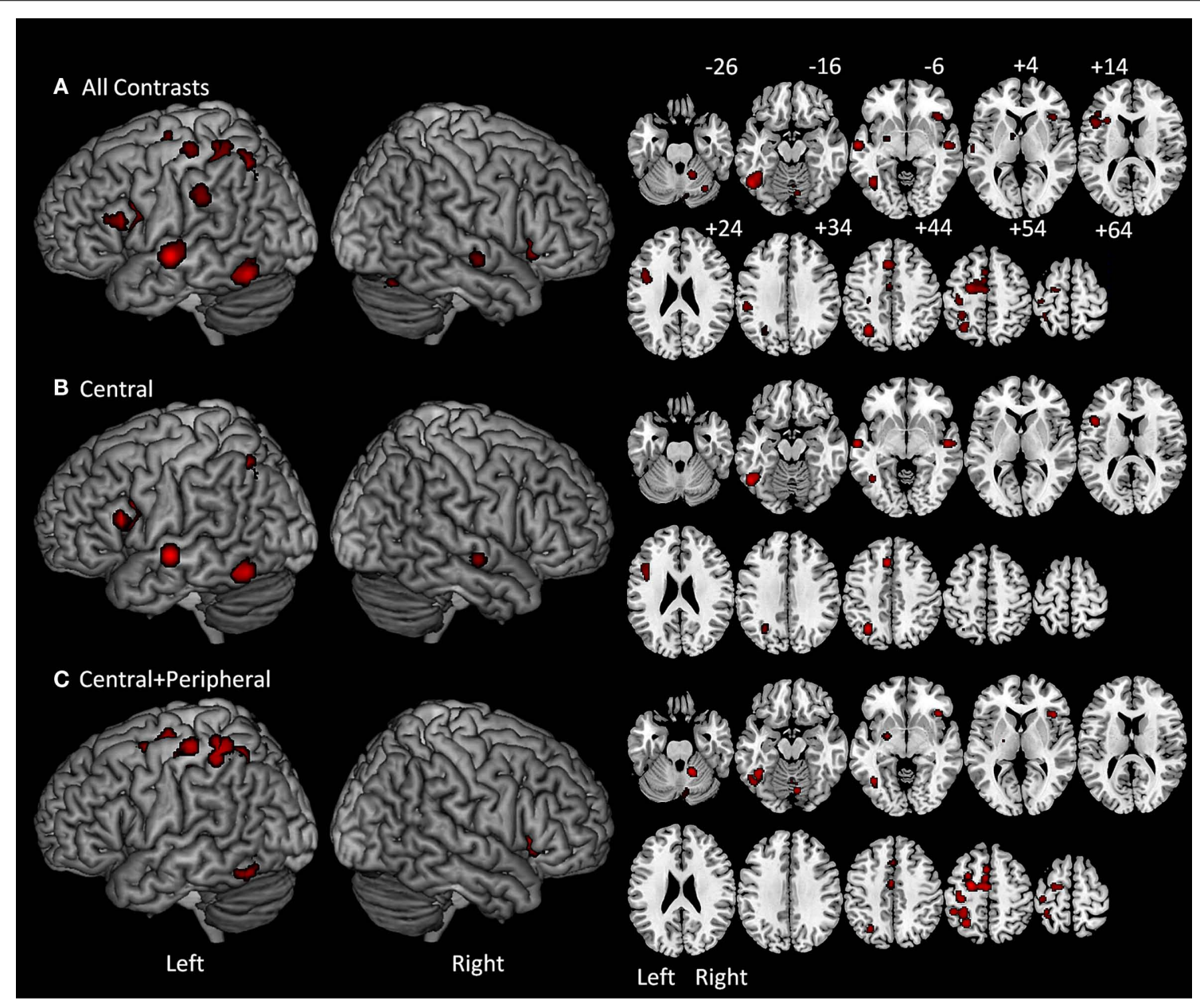

FIGURE 2 | On the left are ALE meta-analysis results projected on a standard rendered template brain and on the right are the corresponding axial slices from $z-\mathbf{2 6}$ to $+\mathbf{6 4}$ in $\mathbf{1 0} \mathrm{mm}$ increments. (A) All-contrasts meta-analysis included all of the tasks referenced in Table 1. (B)
Central-contrasts meta-analysis included only the tasks that involved central, but not peripheral written production processes. (C) Central + peripheral contrast meta-analysis included all of the tasks that involved both central and peripheral written production processes. that extended into the SMA and middle frontal gyrus. Additionally, there was a cluster in the left precentral gyrus that extended posteriorly into the postcentral gyrus. In the parietal lobe there was a large cluster in the superior parietal lobule/intraparietal sulcus. In the occipitotemporal region there was a large cluster within the FG. Sub-cortically, there was a cluster in the left putamen. In the right hemisphere, two clusters were identified in the cerebellum.

\section{ANALYSIS 4: COMPARISON OF C + P VS. C}

The subtraction analysis allowed us to identify locations that were significant for the $\mathrm{C}+\mathrm{P}$ but not the $\mathrm{C}$ analysis. The results revealed three significant clusters with their corresponding peaks and three additional sub-peaks. One cluster was in the left precentral gyrus and extended into the superior frontal sulcus. A second cluster was just posterior to the first, but in the postcentral gyrus. The third cluster was in the left anterior IPS extending dorsally into the superior parietal lobule and ventrally into the SMG (BA40). (Table 4; Figure 3).

\section{DISCUSSION}

This study provides the first quantitative meta-analysis of functional neuroimaging studies of written word production. The analysis is based on 11 studies of alphabetic languages (total $N=146$ ) consisting of a total of 17 experimental contrasts that had been designed to isolate the Central or Central + Peripheral processes of word spelling. Three ALE analyses were carried out. One involved the complete set of 17 contrasts; two others were applied to subsets of contrasts with the goal of distinguishing neural substrates supporting Central from Peripheral processes. In addition a subtraction analysis was performed in order to directly contrast the two subset analyses to identify areas associated specifically with peripheral processes of written word production.

The ALE analysis that considered the full set of 17 contrasts identified significant activation locations in a distributed set of primarily left hemisphere regions that have been previously associated with spelling such as the IFG, superior frontal gyrus/superior frontal sulcus (SFG/SFS), SPL/IPS, 
Table 3 | Results for the central and the central + peripheral ALE meta-analyses.

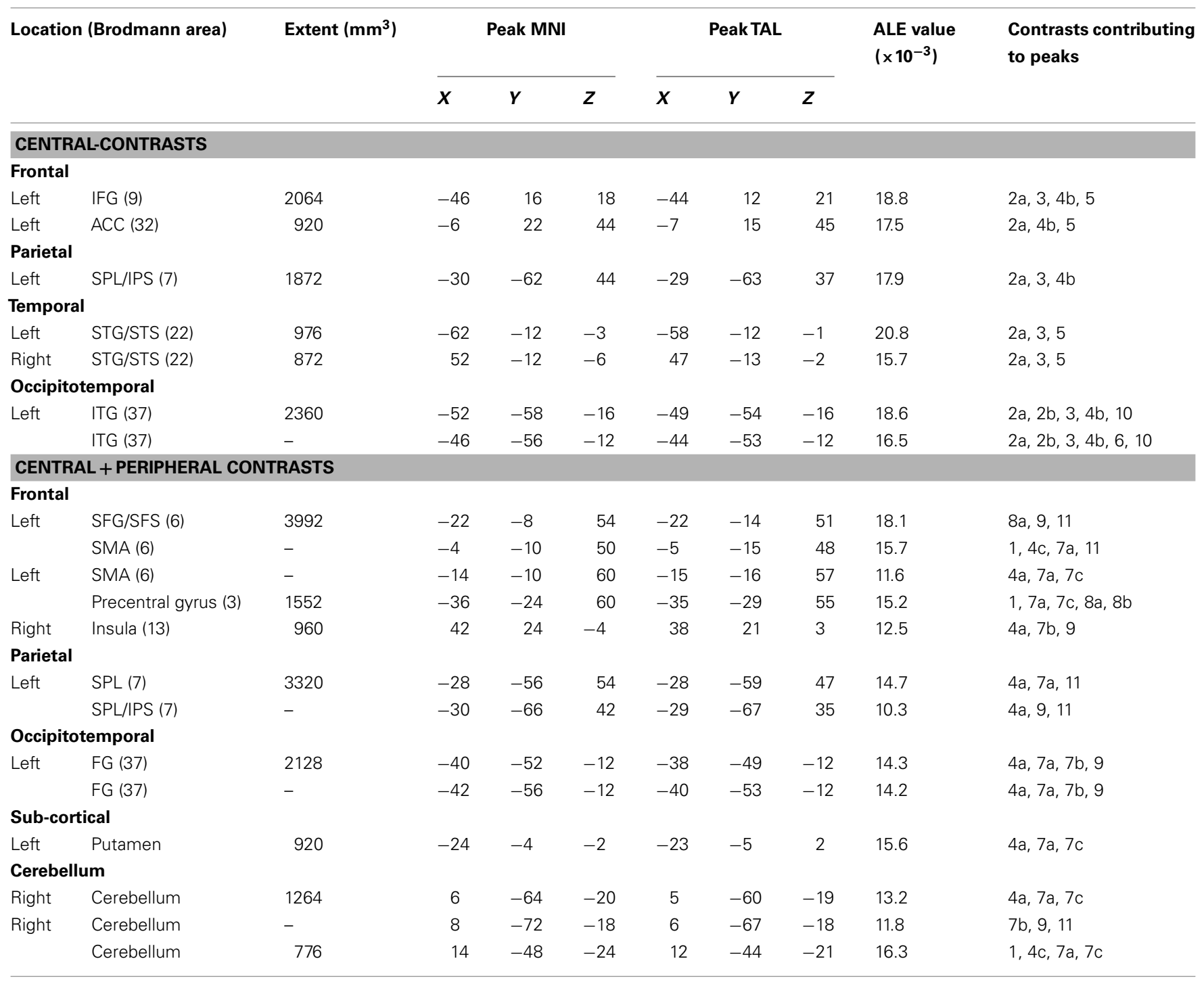

STG/STS, FG, and ITG. In addition to confirming reports from the existing lesion and functional neuroimaging literatures, this analysis provided the most likely specific location of each of these sites. Among the results there are two findings that are particularly noteworthy. We briefly mention them here and discuss them more fully below. First, the analysis revealed that the regions with the highest correspondences across studies were in the left FG/ITG (observed in 11 of the 17 contrasts) and left IFG (observed in 8 of the 17 contrasts). Second, although the lesion literature has traditionally identified the AG as a key site within the written word production network, the meta-analysis did not identify the AG as a consistent activation site. Instead, the analysis identified a region just superior and medial to the AG in the left posterior IPS.

Additional analyses allowed further clarification of the functional roles of the identified regions, in particular whether they were associated with central vs. peripheral written language processes. Areas most consistently involved with central processes were: the left FG/ITG, IFG, and posterior IPS. Peripheral processes, as identified through the subtraction of ALEs, were most robustly associated with foci within the left SFG/SFS, precentral gyrus, postcentral gyrus and SPL. We discuss these results in the sections below within the context of both the neuroimaging and lesion literatures on spelling (Figure 1); we also discuss these findings in relation to the reading literature when appropriate. This discussion highlights points of increasing convergence across the various literatures and also identifies controversies and topics to be pursued in future research.

\section{THE CENTRAL PROCESSES OF SPELLING Fusiform gyrus/inferior temporal gyrus}

One of the most salient and robust findings of the All-Contrasts analysis is that the left FG/ITG region (with three separate MNI peaks at $-50,-60,-16 ;-44,-56,-12$; and $-42,-58,-6)$ had one of the highest ALE values generated in the analysis, as well as the greatest number (11) of contributing contrasts. Thus, despite the very considerable diversity in experimental tasks and contrasts, 
this region was consistently identified as contributing to spelling, and more specifically to the central processes in spelling.

In terms of cognitive processes, this region has been most frequently associated with Orthographic LTM. This is supported by a number of lesion studies (Rapp and Caramazza, 1997; Rapcsak and Beeson, 2004; Philipose et al., 2007; Tsapkini and Rapp, 2010) reporting that lesions to this area resulted in the behavioral profile expected from damage to the Orthographic LTM system. This profile includes difficulties in spelling lower frequency irregular words (i.e., words with atypical PG mappings, e.g., yacht), in the face of accurate spelling of regular words and pseudowords. Consistent with the association of this region with Orthographic LTM, recent fMRI studies have found lexical frequency effects in this region, with larger BOLD responses observed for low vs. high frequency words (Rapp and Lipka, 2011; Rapp and Dufor, 2011). Thus, the lesion and neuroimaging spelling literatures are consistent in indicating that this region plays some role in Orthographic LTM.

Table 4 | Central + peripheral $(\mathbf{C}+\mathrm{P})>$ central $(\mathrm{C})$ contrasts meta-analysis.

\begin{tabular}{|c|c|c|c|c|c|c|c|}
\hline \multirow{2}{*}{$\begin{array}{l}\text { Location } \\
\text { (Brodmann area) }\end{array}$} & \multirow{2}{*}{$\begin{array}{l}\text { Extent } \\
\left(\mathrm{mm}^{3}\right)\end{array}$} & \multicolumn{3}{|c|}{ Peak MNI } & \multicolumn{3}{|c|}{ Peak TAL } \\
\hline & & $x$ & $Y$ & $Z$ & $x$ & $Y$ & $Z$ \\
\hline
\end{tabular}

\begin{tabular}{|c|c|c|c|c|c|c|c|c|}
\hline \multicolumn{9}{|c|}{$(C+P)>C$} \\
\hline \multicolumn{9}{|c|}{ Frontal } \\
\hline Left & $\begin{array}{l}\text { Precentral } \\
\text { gyrus (6) }\end{array}$ & 256 & -24 & -11 & 64 & -24 & -17 & 60 \\
\hline Left & SFG/SFS (6) & - & -26 & -4 & 62 & -26 & -11 & 59 \\
\hline \multicolumn{9}{|c|}{ Parietal } \\
\hline Left & $\begin{array}{l}\text { Postcentral } \\
\text { gyrus (3) }\end{array}$ & 272 & -39 & -27 & 63 & -38 & -32 & 57 \\
\hline Left & $\begin{array}{l}\text { Postcentral } \\
\text { gyrus (3) }\end{array}$ & & -34 & -27 & 58 & -33 & -32 & 53 \\
\hline Left & SPL/IPS (7) & 1744 & -36 & -40 & 57 & -35 & -44 & 51 \\
\hline Left & SPL/IPS (7) & - & -33 & -46 & 53 & -32 & -49 & 47 \\
\hline
\end{tabular}

In addition, from the time of the earliest investigations of written language, the left occipitotemporal cortex has being considered to be critical for reading words (Dejerine, 1892). Since then, numerous functional neuroimaging and lesion studies have confirmed the association of the left mid-FG (bordering on the ITG), with visual word processing in literate individuals (Chialant and Caramazza, 1998; Cohen et al., 2000, 2002; McCandliss et al., 2003; Kronbichler et al., 2004; Gaillard et al., 2006; Sheldon et al., 2008; Dehaene et al., 2010). These findings are also generally consistent with the visual object processing literature which includes the left occipitotemporal cortex as part of the ventral visual system of areas selective to common complex visual objects such as faces or body parts (Kanwisher et al., 1997; Schwarzlose et al., 2005). While the specific role of this region in reading is debated (Price and Devlin, 2003), there is evidence, similar to that found for spelling, that this region is sensitive to the lexical frequency of words (Kronbichler et al., 2004) and it has been designated by some investigators as the storage site of the orthographic word forms used in reading (Glezer et al., 2009).

Although findings from the spelling and reading literatures suggests that both skills share neural substrates in the left occipitotemporal cortex, this claim is most clearly established by studies that examine both spelling and reading within the same individuals. In fact, four recent fMRI studies determined that there was overlapping activation for spelling and reading in the left FG/ITG (Cho et al., 2009; Purcell et al., 2011; Rapp and Lipka, 2011; Rapp and Dufor, 2011). These functional neuroimaging findings are also supported by studies of acute and chronic lesions reporting that lesions or hypoperfusion to the left FG/ITG can impair both spelling and reading in a similar manner (Philipose et al., 2007; Tsapkini and Rapp, 2010).

In sum, the finding of overlap within the FG/ITG region for both reading and spelling in both lesion and neuroimaging literatures, as well as the sensitivity of the region to word frequency, are consistent with some role for this region in the Orthographic LTM system.

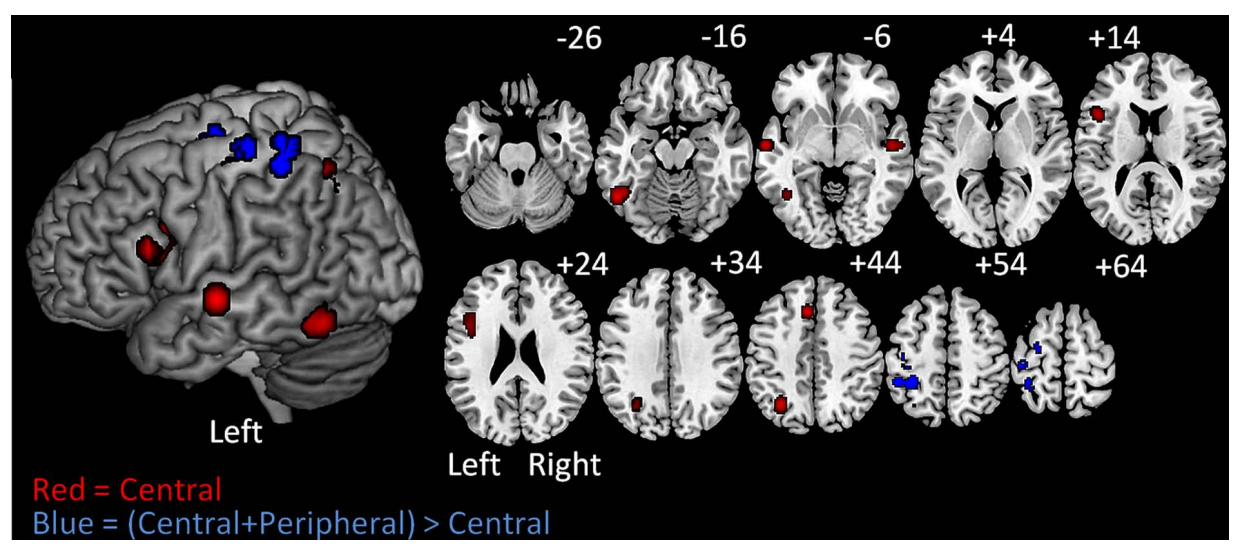

FIGURE 3 | On the left are ALE Meta-analysis results projected on a standard rendered template brain and on the right are the corresponding axial slices from $z-26$ to +64 in $10 \mathrm{~mm}$ increments. In red are the clusters corresponding to the central-contrast analysis. These clusters correspond to the central processes of written word production. In blue are the clusters identified in the (central + peripheral) > central-contrast analysis. These clusters correspond to peripheral processes of written production. 


\section{Left inferior frontal gyrus}

The overall ALE analysis identified a high activation likelihood region within the left IFG (with MNI peaks at: $-46,16,18$ and -44 , $6,28)$ that was supported by eight contrasts, making this region the second most consistently observed across studies. The peaks of this cluster are in the posterior part of the IFG (opercularis), with the cluster extending into the precentral sulcus. This neuroanatomical region has been referred to by some researchers as the Inferior Frontal Junction, IFJ (Brass and Von Cramon, 2002; Brass et al., 2005; Derrfuss et al., 2005).

Damage to this area of cortex is often associated with written and spoken language deficits which is unsurprising considering this region is located within the dorsal extent of Broca's area. More specifically, with regard to acquired dysgraphia in acute stroke, hypoperfusion to the left IFG has been associated with impairments in accessing Orthographic LTM for spelling (Hillis et al., 2002; Hillis and Rapp, 2004). Also consistent with a role for this region in Orthographic LTM, Rapp and Dufor (2011) found the region to be sensitive to word frequency, showing stronger BOLD response to low vs. high frequency words. However, it should be noted that there are studies of chronic stroke associating damage to this region with deficits affecting PG conversion processes (Henry et al., 2007).

With regard to reading, like the FG/ITG region, the posterior IFG is often found to be active in studies of reading (e.g., Fiez and Petersen, 1998; Price, 2000; Turkeltaub et al., 2002; Joubert et al., 2004; Bolger et al., 2005). Furthermore, three of the neuroimaging studies that have considered both reading and spelling in the same individuals reported overlap between spelling and reading in this region (Purcell et al., 2011; Rapp and Lipka, 2011; Rapp and Dufor, 2011). This latter finding suggests a role for this region in the central processes of spelling.

Specific interpretation of the functional role of the posterior IFG in spelling is complicated because activations in this general region are reported in a large number of studies across quite disparate skill domains. For example, Brass and Von Cramon (2002) suggested that the IFJ is involved in cognitive control (see Rapp and Lipka, 2011 for discussion in the context of spelling). Worth noting is that even within the All-Contrasts meta-analysis, we found heterogeneity in this region with six of the contrasts contributing to this area coming from the Central-only group of studies and only two from the Central + Peripheral group (although the contrasts in this latter group must also draw on central spelling processes). This heterogeneity may reflect the sensitivity of this region to the specific task demands and the particular features of the tasks used in the contrasts. One interpretation, which falls generally within a cognitive control account, is that activation in the left IFG for written language processing is not associated directly with Orthographic LTM, but rather with the coordination of activity in more posterior regions (i.e., in temporal or parietal cortex) that are, themselves, more directly involved in processing the stored representations of written language (Mesulam, 1998; Booth et al., 2002; Bitan et al., 2005). This interpretation suggests that the left IFG/IFJ's participation in orthographic processing may primarily consist of its contribution to the processes of orthographic lexical selection and retrieval, rather than storage. This idea is supported by the findings in spoken word production studies which report that the IFJ/IFG region is associated with lexical selection in speaking (Martin et al., 1994; ThompsonSchill et al., 1997).This proposal is further elaborated by Kan and Thompson-Schill (2004) who suggested that biased competition processes (Desimone and Duncan, 1995) draw on this neuroanatomical region when there is competition for selection in visual, lexical, or conceptual domains. That is, while storage of orthographic forms may reside in FG/ITG regions, lexical selection and the resolution of competition at orthographic, lexical, conceptual and/or phonological levels, in spelling and reading, may rely on the left IFJ.

\section{The angular gyrus controversy}

The left angular gyrus (AG) was one of the first regions associated with central processes of spelling and reading (Dejerine, 1892). The region has continued to be associated with literacy and developmental dyslexia (Horwitz et al., 1998; Rumsey et al., 1999; Pugh et al., 2000; Carreiras et al., 2009). The specific function of this region with regard to reading is unclear, however, with some researchers positing that it is part of a larger dorsal parietotemporal region which plays a role in grapheme-phoneme conversion in reading (e.g., Pugh et al., 2000), or that it contributes to lexical processing associated with reading high frequency words (e.g., Joubert et al., 2004). The function of this region with regard to written production is also unclear. Although the left AG has continued to be associated with acquired dysgraphia, there is no clear consensus regarding its functional role in writing. For instance, some studies have linked impairments in irregular word spelling to the left AG suggesting it is involved in Orthographic LTM (Beauvois and Derouesne, 1981; Roeltgen and Heilman, 1984), while others have found it to be associated with deficits to sub-lexical PG conversion spelling processes (Hillis et al., 2002; Sheldon et al., 2008).

Conflicting with the lesion literature findings, the left AG has not been clearly identified in functional neuroimaging studies of spelling. In particular, Beeson et al. (2003) specifically examined the functional activation in a left AG region of interest and found no significant activation for the group analysis, although individual subjects did show some significant effects (Beeson et al., 2003). Interestingly, the left AG has not been consistently identified in the neuroimaging literature of reading either (e.g., Fiez and Petersen, 1998; Turkeltaub et al., 2002; Jobard et al., 2003). Consistent with these previous findings, the meta-analysis did not identify any reliable activation peaks in the AG. This confirms that this region is not reliably identified in functional neuroimaging studies of written word production.

It may be useful to consider these inconsistent reports in light of recent observations that activation in the left AG has been found in neuroimaging studies involving conceptual or semantic processing (Binder et al., 2009; Graves et al., 2009; Seghier et al., 2011). While spelling (as indicated in Figure 1) and reading both engage semantic processes, tasks involving spelling or reading which are not designed to focus the participant's attention on the semantic features of the words may not produce sufficiently consistent activation in semantically related areas, such as the AG, to be detected by experimental paradigms/analysis techniques employed. In sum, the inconsistently observed activation of the AG may be due to low and variable activation of semantic 
processing regions in neuroimaging studies involving spelling or reading tasks that are not focused on semantic processing.

That said, the All-Contrasts analysis did identify a cluster in the posterior IPS which is supported by five experimental contrasts and is located just superior and medial to the left AG, in the left posterior IPS (MNI peak: $-30,-60,46$ ). It is important to point out that the posterior IPS is essentially the neuroanatomical demarcation between the superior parietal lobule and the AG and, therefore, that the IPS cluster that we report could be considered to be at the most superior edge of the AG. Importantly, a similar region was observed in both the $\mathrm{C}$-Contrasts and $\mathrm{C}+\mathrm{P}$ Contrasts separately suggesting that this region plays a role in central spelling processes.

If we consider cognitive processes that have been associated with the SPL/IPS region specifically, we find that damage to superior parietal cortex, entirely, or largely sparing the $A G$, has been associated with spelling deficits, specifically ones affecting Orthographic WM (Miceli et al., 2004; Buchwald and Rapp, 2009). In particular, one of the individuals described by Buchwald and Rapp (2009) had a lesion restricted to the parietal lobe anterior to the AG. Furthermore, also consistent with a role for this region in Orthographic WM is the finding reported by Rapp and Dufor (2011) of sensitivity of the BOLD response in this area to word length in written word production.

One way to reconcile the current body of functional neuroimaging and the lesion findings implicating the left $A G$, is to assume that the IPS region is, in fact, the critical region for spelling and that lesions to the left AG may have also damaged the posterior IPS (e.g., due to the close proximity of these regions) or connections to the IPS. Still another interpretation is that lesions which have previously been associated with the left AG have actually affected a portion of the left inferior temporal lobe or FG that was important for written spelling. This possibility has been suggested in lesion studies examining deficits in spelling (Rapcsak and Beeson, 2004) as well as reading (Epelbaum et al., 2008). One final possibility is that the AG serves to modulate activity in areas related to orthographic processing and that it may not be detectable via classic functional neuroimaging measures utilized in the studies considered in this meta-analysis, but instead can be observed via anatomical or functional connectivity measures. This is supported by recent work which reported gray matter increases in the bilateral AG as well as white matter increases in the splenium of the corpus callosum (which serves to functionally connect the bilateral angular gyri)were associated with learning to read and write as an adult (Carreiras et al., 2009). These varied interpretations indicate that much further research is needed in order to more clearly discern the relevance of the AG to written spelling.

\section{Other perisylvian regions: SMG and STG/STS}

The remaining left hemisphere cortical sites that were identified in the All-Contrasts analysis and that were not attributed to peripheral processes are the mid left STG/STS (MNI peak: -60, -12, -2) and the SMG (MNI peak: $-52,-32,34)$. Lesions to either the left STG/STS or SMG have typically led to impaired written word production by affecting phonological processing thought to be associated with the PG conversion system. This type of impairment results in difficulties in pseudoword spelling with relatively more intact spelling of both regular and irregular familiar words (Henry et al., 2007; also see Philipose et al., 2007 for parallel deficits in spelling and reading pseudowords subsequent to damage to BA40). Furthermore, the left SMG has been directly implicated in functional neuroimaging studies of Japanese Kana writing (a phonetic written language system) which is thought to rely heavily on PG conversion (Katanoda et al., 2001; Sugihara et al., 2006). Analogous studies in an alphabetic language such as English would involve pseudoword writing, but to date no such neuroimaging study has been performed.

With specific regard to the STG/STS sites, it is worth noting that this was the one cortical area in which bilateral activation was observed. Activation in these sites (especially the right hemisphere location) were supported largely by the specific contrasts used by Booth et al. (2002, 2003b, 2004) involving a task that required subjects to compare the rime spelling of three different auditory words. This task, unlike many of the others examined, involved considerable phonological processing and phonological working memory. In this regard it is worth noting that the mid to posterior STG/STS region has been associated in both lesion and neuroimaging studies with phonological processing and phonological deficits (Turkeltaub and Coslett, 2011). Given this, it may not be surprising that this area is involved in PG conversion processes. The similarity and likely close topographic proximity of regions implicated in PG conversion and phonological processes more generally, underscores the importance for future work to be specifically directed at distinguishing spelling-specific from more general phonological processes (e.g., Rapcsak et al., 2009).

\section{THE PERIPHERAL PROCESSES OF SPELLING}

A subtraction of the ALE maps of the Central from the Central + Peripheral contrasts was designed to reveal the locations most likely to have peak activations associated with the peripheral processes of spelling: those processes that are involved with the format-specific production of spelling knowledge (see Figure 1). It is worth making two points before continuing. First, for almost all of the contrasts we considered, there were control conditions designed to "subtract out" the most peripheral aspects of the motor responses produced in these tasks, with the goal of more clearly identifying spelling-specific peripheral processes. Although these contrast conditions may have been more or less successful in accomplishing this goal, it is for this reason that we will not focus on identifying or discussing general motor processes and substrates. Second, although there are empirical and computational reasons to assume that there are multiple components involved in the peripheral aspects of spelling (as depicted in Figure 1 and discussed in the Introduction), relatively little work has been carried out to differentiate them or their neural substrates. Thus, the discussion of the results in this section will be far more limited than was the discussion of the central processes of spelling.

The regions identified as being primarily associated with peripheral processes were: the left precentral gyrus and SFG/SFS, the left postcentral gyrus and left SPL/IPS. In addition, there were other motor related regions, such as the left SMA and the right cerebellum, that were significant in the All-Contrasts 
meta-analysis and were supported by contrasts that involved handwriting or typing output (some of which did not include motor baselines). All of these areas have been implicated in the lesion literature as being important for writing. Some have been linked specifically to writing such as the left SFG/SFS and the SPL/IPS, while others have been associated with motor processes more generally, such as the left SMA, left postcentral gyrus and the right cerebellum (see Gerloff et al. (1997) for discussion regarding the SMA). In subsequent sections we focus our discussion on the left SFG/SFS and the SPL/IPS.

\section{The precentral gyrus and superior frontal gyrus/sulcus}

The subtraction analysis designed to identify peripheral writing substrates identified a high ALE region centered in the left precentral gyrus (MNI peak: $-24,-11,64)$ and extending anteriorly into the SFG/SFS (MNI peak: $-26,-4,62$ ).

In the lesion literature, the conversion of graphemic representations to motor commands has been associated with a region in the left posterior middle frontal gyrus and SFG (BA6) often referred to in the clinical neuroscience literature as Exner's area. For handwriting, this region has primarily been associated with the translation of orthographic representations into letter shapes (i.e., allographic processing; Exner, 1881; Ritaccio et al., 1992; Tohgi et al., 1995; Lubrano et al., 2004). Interestingly a recent fMRI study of Japanese Kana handwriting identified a peak in the left SFS (MNI peak: -28, -9, 54; Sugihara et al., 2006) that is within $7 \mathrm{~mm}$ of the SFS peak identified in our Central + Peripheral meta-analysis (MNI peak: $-22,-8,54$ ). These results suggest that this region may be associated with processes common to both alphabetic and syllabic writing systems such as Kana. Additionally, upon further examination of the SFG/SFS peak from the Central + Peripheral analysis from Table 3, we find that this peak was associated with two handwriting tasks and one keyboard typing study, thus suggesting that it may play a role in the conversion of graphemic representations to motor commands regardless of whether the word is handwritten or typed. Based on these findings it seems that, although the left SFG/SFS is consistently associated with written motor production, the specific functional role of this region in the conversion of graphemes to motor commands needs to be examined further with regard to whether or not there is specificity for output modality (i.e., handwriting or typing).

Another possibility regarding the function of the precentral/SFG/SFS region is that it plays a critical role in Orthographic WM. This was proposed in a recent fMRI study comparing activations from the spelling of longer as compared to shorter words (matched for writing times and motor output) which reported length dependent activation in both the left SFS and SPL (Rapp and Dufor, 2011). These authors argued that not only was the SFS finding indicative of Orthographic WM functions for this frontal region, but that this claim would explain the findings that the area is recruited for spelling across modalities of output (handwriting and typing), as both output formats should rely on a common Orthographic WM system. Reporting findings consistent with this proposal, Cloutman et al. (2009) carried out a study of individuals in the first $48 \mathrm{~h}$ after left hemisphere stroke onset, with one group showing clear signs of Orthographic WM impairment while the other had graphemic buffer sparing. They found that, among other regions, the left premotor cortex was reliably associated with graphemic buffer damage.

The inconsistency that the Rapp and Dufor (2011) proposal creates is that Orthographic WM is considered to be a component of central spelling, yet it was identified by the meta-analysis as being especially associated with peripheral processes. However, it is important to note that Orthographic WM is situated at the interface between central and peripheral processing and may actually interact with peripheral component processes in a manner which may make it difficult to distinguish between central and peripheral processes that are in the same or neighboring regions. Further work will need to be done to better characterize the functional role of the left SFS with regard to its role in Orthographic WM as compared to the more peripheral processes of written production. In addition, if there turns out to be a reliable relationship between Orthographic WM and the posterior SPL/IPS regions (as suggested in an earlier section), the specific roles of the frontal and parietal areas in Orthographic WM will also need to be examined. In that regard it is interesting to note that there have been several proposals of multiple cognitive components of Orthographic WM, much like the multi-component view of other WM systems (see Rapp and Kong, 2002; Kan et al., 2006). Presumably, different components of Orthographic WM would draw on different neural substrates and may explain the sensitivity of both SFS and SPL to the length of words being spelled.

\section{Superior parietal lobule}

Aside from the generation of graphemic motor commands, writing requires the generation of the correct sequences of motor commands. The left SPL in particular has been associated with sequence production in written language. For instance, damage to left SPL has be associated with apraxic agraphia, a disorder characterized by deficits in the generation of correct sequences of movements required for handwriting (Alexander et al., 1992; Sakurai et al., 2007). In support of these findings we identified a left anterior SPL/IPS cluster which was shown to be associated with the peripheral but not central processes of written production (MNI peak: $-36,-40,57)$. This peak is notably anterior to the one identified in the Central-contrasts analysis (MNI peak: $-30,-62$, 44) which suggests that there may be a distinction between certain peripheral processes that draw on the more anterior SPL and central processes (e.g., Orthographic WM) that make use of the more posterior SPL/IPS region.

The left SPL has also been associated with the learning and generation of complex motor sequences (Haaland et al., 2004) as well as with the integration of the body-schema with allo- and egocentric frames of reference (Neggers et al., 2006). Other work has shown that the left SPL, in particular the region including the IPS, is topographically organized such that the more anterior portion is associated with the coordination of body movements in space (e.g., grasping and pointing) and the more posterior regions are associated with calculation and eye saccades (Simon et al., 2002). Understanding the relationship amongst the cognitive requirements of writing and these other tasks and, in turn, the specific neural substrates that support these cognitive processes will be an important research direction. 


\section{SUMMARY AND CONCLUSION}

This study provides the first quantitative review and synthesis of findings from functional neuroimaging studies of written language production. The analyses carried out identify a network of left hemisphere frontal, parietal, and temporal sites that are reliably and consistently associated with written word production, providing estimates of the most likely locations of peak activations within these areas. Furthermore, these analyses provide a coherent assignment of these locations to central or peripheral processes of spelling. The General Discussion relates these sites to more specific cognitive functions by bringing together results from the lesion and neuroimaging literatures. This study sets the stage for future research regarding the neural substrates of written word

\section{REFERENCES}

Alexander, M. P., Fischer, R. S., and Friedman, R. (1992). Lesion localization in apractic agraphia. Arch. Neurol. 49, 246-251.

Beauvois, M. F., and Derouesne, J. (1981). Lexical or orthographic agraphia. Brain 104, 21-49.

Beeson, P., Rapcsak, S., Plante, E., Chargualaf, J., Chung, A., Johnson, S., and Trouard, T. (2003). The neural substrates of writing: a functional magnetic resonance imaging study. Aphasiology 17, 647-665.

Binder, J. R., Desai, R. H., Graves, W. W., and Conant, L. L. (2009). Where is the semantic system? A critical review and meta-analysis of 120 functional neuroimaging studies. Cereb. Cortex 19, 2767-2796.

Bitan, T., Booth, J. R., Choy, J., Burman, D. D., Gitelman, D. R., and Mesulam, M. M. (2005). Shifts of effective connectivity within a language network during rhyming and spelling. J. Neurosci. 25, 5397-5403.

Bolger, D. J., Perfetti, C. A., and Schneider, W. (2005). Cross-cultural effect on the brain revisited: universal structures plus writing system variation. Hum. Brain Mapp. 25, 92-104.

Booth, J. R., Burman, D. D., Meyer, J. R., Gitelman, D. R., Parrish, T. B., and Mesulam, M. M. (2002). Functional anatomy of intra- and crossmodal lexical tasks. Neuroimage 16, 7-22.

Booth, J. R., Burman, D. D., Meyer, J. R., Gitelman, D. R., Parrish, T. B., and Mesulam, M. M. (2003a). Relation between brain activation and lexical performance. Hum. Brain Mapp. 19, 155-169.

Booth, J. R., Burman, D. D., Meyer, J. R., Lei, Z., Choy, J., Gitelman, D. R., Parrish, T. B., and Mesulam, M. M. (2003b). Modality-specific and -independent developmental differences in the neural substrate for lexical processing. J. Neurolinguistics 16, 383-405.

Booth, J. R., Burman, D. D., Meyer, J. R., Gitelman, D. R., Parrish, T. B., and Mesulam, M. M. (2004). Development of brain mechanisms for processing orthographic and phonologic representations. J. Cogn. Neurosci. 16, 1234-1249.

Brass, M., Derrfuss, J., Forstmann, B., and Von Cramon, D. Y. (2005). The role of the inferior frontal junction area in cognitive control. Trends Cogn. Sci. (Regul. Ed.) 9, 314-316.

Brass, M., and Von Cramon, D. Y. (2002). The role of the frontal cortex in task preparation. Cereb. Cortex 12, 908-914.

Buchwald, A., and Rapp, B. (2009). Distinctions between orthographic long-term memory and working memory. Cogn. Neuropsychol. 26, 724-751.

Burt, J. S., and Fury, M. B. (2000). Spelling in adults: the role of reading skills and experience. Read. Writ. $13,1-30$.

Burt, J. S., and Tate, H. (2002). Does a reading lexicon provide orthographic representations for spelling? J. Mem. Lang. 46, 518-543.

Caramazza, A., and Hillis, A. E. (1990). Spatial representation of words in the brain implied by studies of a unilateral neglect patient. Nature 346, 267-269.

Carreiras, M., Seghier, M. L., Baquero, S., Estevez, A., Lozano, A., Devlin, J. T., and Price, C. J. (2009). An anatomical signature for literacy. Nature 461, 983-986.

Chialant, D., and Caramazza, A. (1998). Perceptual and lexical factors in a case of letter-by-letter reading. Cogn. Neuropsychol. 15, 167-201.

Cho, H., Rapcsak, S. Z., and Beeson, P. M. (2009). Evidence for a common neural substrate of orthographic processing during reading and spelling. Neurobiology of Language Conference, Chicago, IL.

production by identifying the literature's most reliable findings and by highlighting critical questions regarding the neural basis of written language productions that still need to be addressed by future studies.

\section{ACKNOWLEDGMENTS}

This work was supported by NIDCD006740 (Brenda Rapp), NICHD R01HD056107 (Guinevere F. Eden), NIDCD (F31DC009545) (Jeremy J. Purcell), NSF SBE 0541953 (Jeremy J. Purcell and Guinevere F. Eden), and the American Academy of Neurology Foundation Clinical Research Training Fellowship (Peter E. Turkeltaub). We would like to thank Drs Pelagie Beeson and Olivier Dufor for providing additional data for these analyses.

Cloutman, L., Gingis, L., Newhart, M., Davis, C., Heidler-Gary, J., Crinion, J., and Hillis, A. E. (2009). A neural network critical for spelling. Ann. Neurol. 66, 249-253.

Cohen, L., Dehaene, S., Naccache, L., Lehericy, S., Dehaene-Lambertz, G., Henaff, M. A., and Michel, F. (2000). The visual word form area: spatial and temporal characterization of an initial stage of reading in normal subjects and posterior splitbrain patients. Brain $123(\mathrm{Pt} 2)$, 291-307.

Cohen, L., Jobert, A., Le Bihan, D., and Dehaene, S. (2004). Distinct unimodal and multimodal regions for word processing in the left temporal cortex. Neuroimage 23, 1256-1270.

Cohen, L., Lehericy, S., Chochon, F. Lemer, C., Rivaud, S., and Dehaene, S. (2002). Language-specific tuning of visual cortex? Functional properties of the visual word form area. Brain 125, 1054-1069.

Dehaene, S., Pegado, F., Braga, L. W., Ventura, P., Nunes Filho, G., Jobert, A., Dehaene-Lambertz, G., Kolinsky, R., Morais, J., and Cohen, L. (2010). How learning to read changes the cortical networks for vision and language. Science 330, 1359-1364.

Dejerine, J. (1892). Contribution a l'étude anatomopathologique et clinique des différentes variétés de cécité verbale. Mem. Soc. Biol. 4, 61-90.

Denes, G., Signorini, M., and Volpato, C. (2005). Post graphemic impairments of writing: the case of micrographia. Neurocase 11, 176-181.

Derrfuss, J., Brass, M., Neumann, J., and Von Cramon, D. Y. (2005). Involvement of the inferior frontal junction in cognitive control: meta-analyses of switching and Stroop studies. Hum. Brain Mapp. 25, 22-34.

Desimone, R., and Duncan, J. (1995). Neural mechanisms of selective visual attention. Annu. Rev. Neurosci. 18, 193-222.
Eickhoff, S. B., Laird, A. R., Grefkes, C., Wang, L. E., Zilles, K., and Fox, P. T. (2009). Coordinate-based activation likelihood estimation metaanalysis of neuroimaging data: a random-effects approach based on empirical estimates of spatial uncertainty. Hum. Brain Mapp. 30, 2907-2926.

Eickhoff, S. B., Stephan, K. E., Mohlberg, H., Grefkes, C., Fink, G. R., Amunts, K., and Zilles, K. (2005). A new SPM toolbox for combining probabilistic cytoarchitectonic maps and functional imaging data. Neuroimage 25, 1325-1335.

Ellis, A. W. (1979). Slips of the pen. Visible Lang. 13, 265-282.

Ellis, A. W. (1982). "Spelling and writing (and reading and speaking)," in Normality and Pathology in Cognitive Functions, ed. A. W. Ellis (New York: Academic Press), 113-143.

Ellis, A. W., and Young, A. W. (1988). Human Cognitive Neuropsychology. London: Lawrence Erlbaum Associates.

Epelbaum, S., Pinel, P., Gaillard, R., Delmaire, C., Perrin, M., Dupont, S., Dehaene, S., and Cohen, L. (2008). Pure alexia as a disconnection syndrome: new diffusion imaging evidence for an old concept. Cortex 44 , 962-974.

Exner, S. (1881). Untersuchungen über die Lokalisation der Functionen in der Grosshirnrinde des Menschen. Wien: W. Braumuller.

Fiez, J. A., and Petersen, S. E. (1998). Neuroimaging studies of word reading. Proc. Natl. Acad. Sci. U.S.A. 95, 914-921.

Fournier Del Castillo, M. C., Maldonado Belmonte, M. J., RuizFalco Rojas, M. L., Lopez Pino, M. A., Bernabeu Verdu, J., and Suarez Rodriguez, J. M. (2011). Cerebellum atrophy and development of a peripheral dysgraphia: a paediatric case. Cerebellum 9, 530-536. 
Gaillard, R., Naccache, L., Pinel, P., Clemenceau, S., Volle, E., Hasboun, D., Dupont, S., Baulac, M., Dehaene, S., Adam, C., and Cohen, L. (2006). Direct intracranial, FMRI, and lesion evidence for the causal role of left inferotemporal cortex in reading. Neuron 50, 191-204.

Gerloff, C., Corwell, B., Chen, R., Hallett, M., and Cohen, L. G. (1997). Stimulation over the human supplementary motor area interferes with the organization of future elements in complex motor sequences. Brain 120(Pt 9), 1587-1602.

Glezer, L. S., Jiang, X., and Riesenhuber, M. (2009). Evidence for highly selective neuronal tuning to whole words in the "visual word form area." Neuron 62, 199-204.

Graves, W. W., Desai, R., Humphries, C., Seidenberg, M. S., and Binder, J. R. (2009). Neural systems for reading aloud: a multiparametric approach. Cereb. Cortex 20, 1799-1815.

Haaland, K. Y., Elsinger, C. L., Mayer, A. R., Durgerian, S., and Rao, S. M. (2004). Motor sequence complexity and performing hand produce differential patterns of hemispheric lateralization. J. Cogn. Neurosci. 16, 621-636.

Henry, M. L., Beeson, P. M., Stark, A. J., and Rapcsak, S. Z. (2007). The role of left perisylvian cortical regions in spelling. Brain Lang. 100, 44-52.

Hillis, A., Kane, A., Tuffiash, E., Beauchamp, N., Barker, P., Jacobs, M., and Wityk, R. (2002). Neural substrates of the cognitive processes underlying spelling: evidence from MR diffusion and perfusion imaging. Aphasiology 16, 425-438.

Hillis, A., and Rapp, B. (2004). "Cognitive and neural substrates of written language: comprehension and production," in The Cognitive $\mathrm{Neu}$ rosciences III, ed. M. S. Gazzaniga (Cambridge, MA: The MIT press), 775-787.

Holmes, C. J., Hoge, R., Collins, L., Woods, R., Toga, A. W., and Evans, A. C. (1998). Enhancement of MR images using registration for signal averaging. J. Comput. Assist. Tomogr. 22, 324-333.

Horwitz, B., Rumsey, J. M., and Donohue, B. C. (1998). Functional connectivity of the angular gyrus in normal reading and dyslexia. Proc. Natl. Acad. Sci. U.S.A. 95, 8939-8944.

Jobard, G., Crivello, F., and TzourioMazoyer, N. (2003). Evaluation of the dual route theory of reading: a metanalysis of 35 neuroimaging studies. Neuroimage 20, 693-712.

Joubert, S., Beauregard, M., Walter, N., Bourgouin, P., Beaudoin, G., Leroux,
J. M., Karama, S., and Lecours, A. R. (2004). Neural correlates of lexical and sublexical processes in reading. Brain Lang. 89, 9-20.

Kan, I. P., Biran, I., Thompson-Schill, S. L., and Chatterjee, A. (2006). Letter selection and letter assembly in acquired dysgraphia. Cogn. Behav. Neurol. 19, 225-236.

Kan, I. P., and Thompson-Schill, S. L. (2004). Selection from perceptual and conceptual representations. Cogn. Affect. Behav. Neurosci. 4, 466-482.

Kanwisher, N., Mcdermott, J., and Chun, M. M. (1997). The fusiform face area: a module in human extrastriate cortex specialized for face perception. J. Neurosci. 17, 4302-4311.

Katanoda, K., Yoshikawa, K., and Sugishita, M. (2001). A functional MRI study on the neural substrates for writing. Hum. Brain Mapp. 13, 34-42.

Kawahata, N., Nagata, K., and Shishido, F. (1988). Alexia with agraphia due to the left posterior inferior temporal lobe lesion - neuropsychological analysis and its pathogenetic mechanisms. Brain Lang. 33, 296-310.

Kronbichler, M., Hutzler, F., Wimmer, H., Mair, A., Staffen, W., and Ladurner, G. (2004). The visual word form area and the frequency with which words are encountered: evidence from a parametric fMRI study. Neuroimage 21, 946-953.

Lancaster, J. L., Tordesillas-Gutierrez, D., Martinez, M., Salinas, F., Evans, A., Zilles, K., Mazziotta, J. C., and Fox, P. T. (2007). Bias between MNI and Talairach coordinates analyzed using the ICBM-152 brain template. Hum. Brain Mapp. 28, 1194-1205.

Lubrano, V., Roux, F. E., and Demonet, J. F. (2004). Writing-specific sites in frontal areas: a cortical stimulation study. J. Neurosurg. 101, 787-798.

Magrassi, L., Bongetta, D., Bianchini, S., Berardesca, M., and Arienta, C. (2010). Central and peripheral components of writing critically depend on a defined area of the dominant superior parietal gyrus. Brain Res. 1346, 145-154.

Margolin, D. I. (1984). The neuropsychology of writing and spelling: semantic, phonological, motor, and perceptual processes. Q. J. Exp. Psychol. A. 36, 459-489.

Martin, N., Dell, G. S., Saffran, E. M., and Schwartz, M. F. (1994). Origins of paraphasias in deep dysphasia: testing the consequences of a decay impairment to an interactive spreading activation model of lexical retrieval. Brain Lang. 47, 609-660.

Mesulam, M. M. (1998). From sensation to cognition. Brain 121 (Pt 6), 1013-1052.

McCandliss, B. D., Cohen, L., and Dehaene, S. (2003). The visual word form area: expertise for reading in the fusiform gyrus. Trends Cogn. Sci. (Regul. Ed.) 7, 293-299.

McCloskey, M., Macaruso, P., and Rapp, B. (2006). Grapheme-tolexeme feedback in the spelling system: evidence from a dysgraphic patient. Cogn. Neuropsychol. 23, 278-307.

Menon, V., and Desmond, J. E. (2001). Left superior parietal cortex involvement in writing: integrating fMRI with lesion evidence. Brain Res. Cogn. Brain Res. 12, 337-340.

Miceli, G., Capasso, R., Benvegnu, B., and Caramazza, A. (2004). The categorical distinction of vowel and consonant representations: evidence from dysgraphia. Neurocase 10 , 109-121.

Neggers, S. F., Van Der Lubbe, R. H., Ramsey, N. F., and Postma, A. (2006). Interactions between ego- and allocentric neuronal representations of space. Neuroimage 31, 320-331.

Patterson, K. (1986). Lexical but nonsemantic spelling? Cogn. Neuropsychol. 3, 341-367.

Petrides, M., Alivisatos, B., and Evans, A. C. (1995). Functional activation of the human ventrolateral frontal cortex during mnemonic retrieval of verbal information. Proc. Natl. Acad. Sci. U.S.A. 92, 5803-5807.

Philipose, L. E., Gottesman, R. F. Newhart, M., Kleinman, J. T., Herskovits, E. H., Pawlak, M. A., Marsh, E. B., Davis, C., Heidler-Gary, J., and Hillis, A. E. (2007). Neural regions essential for reading and spelling of words and pseudowords. Ann. Neurol. 62, 481-492.

Pramstaller, P. P., and Marsden, C. D. (1996). The basal ganglia and apraxia. Brain 119(Pt 1), 319-340.

Price, C. (2000). The anatomy of language: contributions from functional neuroimaging. J. Anat. 197, 335-359.

Price, C. J., and Devlin, J. T. (2003). The myth of the visual word form area. Neuroimage 19, 473-481.

Price, C. J., Devlin, J. T., Moore, C. J., Morton, C., and Laird, A. R. (2005). Meta-analyses of object naming: effect of baseline. Hum. Brain Mapp. 25, 70-82.

Pugh, K. R., Mencl, W. E., Shaywitz, B. A., Shaywitz, S. E., Fulbright, R. K., Constable, R. T., Skudlarski, P., Marchione, K. E., Jenner, A.
R., Fletcher, J. M., Liberman, A. M., Shankweiler, D. P., Katz, L., Lacadie, C., and Gore, J. C. (2000). The angular gyrus in developmental dyslexia: task-specific differences in functional connectivity within posterior cortex. Psychol. Sci. 11, 51-56.

Purcell, J. J., Napoliello, E. M., and Eden, G. F. (2011). A combined fMRI study of typed spelling and reading. Neuroimage 55, 750-762.

Rapcsak, S., and Beeson, P. (2002). "Neuroanatomical correlates of spelling and writing," in The Handbook of Adult Language Disorders, ed. A. Hillis (New York: Psychology Press), 71-100.

Rapcsak, S. Z., and Beeson, P. M. (2004). The role of left posterior inferior temporal cortex in spelling. Neurology 62, 2221-2229.

Rapcsak, S. Z., Beeson, P. M., Henry, M. L., Leyden, A., Kim, E., Rising, K., Andersen, S., and Cho, H. (2009). Phonological dyslexia and dysgraphia: cognitive mechanisms and neural substrates. Cortex 45 , 575-591.

Rapp, B., and Caramazza, A. (1997). From graphemes to abstract letter shapes: levels of representation in written spelling. J. Exp. Psychol. Hum. Percept. Perform. 23, 1130-1152.

Rapp, B., and Dufor, O. (2011). The neurotopography of written word production: an fMRI investigation of the distribution of sensitivity to word length and frequency. J. Cogn. Neurosci. PMID: 21812571. [Epub ahead of print].

Rapp, B., Epstein, C., and Tainturier, M. J. (2002). The integration of information across lexical and sublexical processes in spelling. Cogn. Neuropsychol. 19, 1-29.

Rapp, B., and Kong, D. (2002). Revealing the component functions of the graphemic buffer. Brain Lang. 83, 112-114.

Rapp, B., and Lipka, K. (2011). The literate brain: the relationship between spelling and reading. J. Cogn. Neurosci. 23, 1-18.

Ritaccio, A. L., Hickling, E. J., and Ramani, V. (1992). The role of dominant premotor cortex and grapheme to phoneme transformation in reading epilepsy. A neuroanatomic, neurophysiologic, and neuropsychological study. Arch. Neurol. 49, 933-939.

Roeltgen, D. P., and Heilman, K. M. (1984). Lexical agraphia. Further support for the two-system hypothesis of linguistic agraphia. Brain 107(Pt 3), 811-827. 
Roeltgen, D. P., and Heilman, K. M. (1985). Review of agraphia and a proposal for an anatomically-based neuropsychological model of writing. Appl. Psycholinguist. 6, 205-229.

Roux, F. E., Dufor, O., Giussani, C., Wamain, Y., Draper, L., Longcamp, M., and Demonet, J. F. (2009). The graphemic/motor frontal area Exner's area revisited. Ann. Neurol. 66, 537-545.

Rumsey, J. M., Horwitz, B., Donohue, B. C., Nace, K. L., Maisog, J. M., and Andreason, P. (1999). A functional lesion in developmental dyslexia: left angular gyral blood flow predicts severity. Brain Lang. 70, 187-204.

Sakurai, Y., Onuma, Y., Nakazawa, G., Ugawa, Y., Momose, T., Tsuji, S., and Mannen, T. (2007). Parietal dysgraphia: characterization of abnormal writing stroke sequences, character formation and character recall. Behav. Neurol. 18, 99-114.

Salthouse, T. A. (1986). Perceptual, cognitive, and motoric aspects of transcription typing. Psychol. Bull. 99, 303-319.

Salthouse, T. A., and Saults, J. S. (1987). Multiple spans in transcription typing. J. Appl. Psychol. 72, 187-196.

Schwarzlose, R. F., Baker, C. I., and Kanwisher, N. (2005). Separate face and body selectivity on the fusiform gyrus. J. Neurosci. 25, 11055-11059.

Seghier, M. L., Fagan, E., and Price, C. J. (2011). Functional subdivisions in the left angular gyrus where the semantic system meets and diverges from the default network. J. Neurosci. 30, 16809-16817.

Shallice, T. (1981). Phonological agraphia and the lexical route in writing. Brain 104, 413-429.

Sheldon, C. A., Malcolm, G. L., and Barton, J. J. (2008). Alexia with and without agraphia: an assessment of two classical syndromes. Can. J. Neurol. Sci. 35, 616-624.

Simon, O., Mangin, J. F., Cohen, L., Le Bihan, D., and Dehaene, S. (2002). Topographical layout of hand, eye, calculation, and language-related areas in the human parietal lobe. Neuron 33, 475-487.

Sugihara, G., Kaminaga, T., and Sugishita, M. (2006). Interindividual uniformity and variety of the "writing center": a functional MRI study. Neuroimage 32, 1837-1849.

Talairach, J., and Tournoux, P. (1988). Co-planar stereotaxic-atlas of the human brain. 3-Dimensional Proportional System: Approach to Cerebral Imaging. New York: Thieme Medical Publishers Inc.

Tanridag, O., and Kirshner, H. S. (1985). Aphasia and agraphia in lesions of the posterior internal capsule and putamen. Neurology 35, 1797-1801.

Thompson-Schill, S. L., D'Esposito, M., Aguirre, G. K., and Farah, M. J. (1997). Role of left inferior prefrontal cortex in retrieval of semantic knowledge: a reevaluation. Proc. Natl. Acad. Sci. U.S.A. 94, 14792-14797.

Tohgi, H., Saitoh, K., Takahashi, S., Takahashi, H., Utsugisawa, K.,
Yonezawa, H., Hatano, K., and Sasaki, T. (1995). Agraphia and acalculia after a left prefrontal (F1, F2) infarction. J. Neurol. Neurosurg. Psychiatr. 58, 629-632.

Tsapkini, K., and Rapp, B. (2010). The orthography-specific functions of the left fusiform gyrus: evidence of modality and category specificity. Cortex 46 185-205.

Turkeltaub, P. E., and Coslett, H. B. (2011). Localization of sublexical speech perception components. Brain Lang. 114, 1-15.

Turkeltaub, P. E., Eden, G. F., Jones, K. M., and Zeffiro, T. A. (2002) Meta-analysis of the functional neuroanatomy of single-word reading: method and validation. Neuroimage 16, 765-780.

Turkeltaub, P. E., Eickhoff, S. B., Laird, A. R., Fox, M., Wiener, M., and Fox, P. (2011). Minimizing withinexperiment and within-group effects in activation likelihood estimation meta-analyses. Hum. Brain Mapp. doi: 10.1002/hbm.21186. [Epub ahead of print].

Tzourio-Mazoyer, N., Landeau, B., Papathanassiou, D., Crivello, F., Etard, O., Delcroix, N., Mazoyer, B. and Joliot, M. (2002). Automated anatomical labeling of activations in SPM using a macroscopic anatomical parcellation of the MNI MRI single-subject brain. Neuroimage 15 , 273-289.

Weingarten, R. (2005). Subsyllabic units in written word production. Writ. Lang. Lit. 8, 43-61.
Weingarten, R., Nottbusch, G., and Will, U. (2004). "Morphemes, syllables and graphemes in written word production," in Multidisciplinary Approaches to Language Production, ed. T. H. Pechmann (Berlin, NY: Mouton de Gruyter), 529-572.

West, L. J., and Sabban, Y. (1982). Hierarchy of stroking habits at the typewriter. J. Appl. Psychol. 67, 370-376.

Conflict of Interest Statement: The authors declare that the research was conducted in the absence of any commercial or financial relationships that could be construed as a potential conflict of interest.

Received: 18 April 2011; accepted: 01 September 2011; published online: 11 October 2011.

Citation: Purcell JJ, Turkeltaub PE, Eden GF and Rapp B (2011) Examining the central and peripheral processes of written word production through metaanalysis. Front. Psychology 2:239. doi: 10.3389/fpsyg.2011.00239

This article was submitted to Frontiers in Language Sciences, a specialty of Frontiers in Psychology.

Copyright (c) 2011 Purcell, Turkeltaub, Eden and Rapp. This is an open-access article subject to a non-exclusive license between the authors and Frontiers Media $S A$, which permits use, distribution and reproduction in other forums, provided the original authors and source are credited and other Frontiers conditions are complied with. 